\title{
Tratamento da consolidação viciosa do rádio distal: Osteotomia corretiva mediante planejamento com prototipagem em impressão 3D*
}

\section{Treatment of Distal Radio Vicious Consolidation: Corrective Osteotomy Through 3D Printing Prototyping}

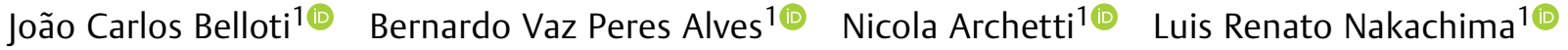 \\ Flavio Faloppa1(1) Marcel Jun Sugawara Tamaoki ${ }^{1 \oplus}$ \\ 1 Disciplina de Cirurgia da Mão, Departamento de Ortopedia e \\ Traumatologia, Escola Paulista de Medicina, Universidade Federal de \\ São Paulo, São Paulo, SP, Brasil

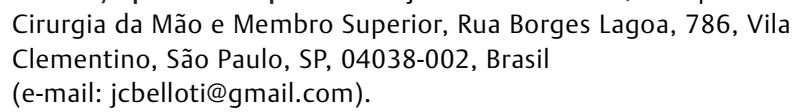
Cirurgia da Mão e Membro Superior, Rua Borges Lagoa, 786, Vila Clementino, São Paulo, SP, 04038-002, Brasil (e-mail: jcbelloti@gmail.com). \\ Endereço para correspondência João Carlos Belloti, Disciplina de
}

Rev Bras Ortop 2021;56(3):384-389.

\section{Resumo \\ Palavras-chave \\ - deformidade do rádio \\ - complicações fraturas do rádio \\ - tomografia por raios $\mathrm{x}$ \\ - impressão tridimensional}

As fraturas da porção distal do rádio estão entre as mais comuns do esqueleto. A consolidação viciosa pode ocorrer em até um terço dessas fraturas e acarretar restrição de movimento e dor no punho, com consequente limitação ou incapacidade laboral. O tratamento desta condição implica em osteotomia corretiva das deformidades, o que necessita de um planejamento pré-operatório criterioso em virtude de sua complexidade tridimensional, notadamente naquelas em que há acometimento articular. Assim, recentemente, tem sido utilizada a prototipagem a partir da reconstrução 3D da tomografia computadorizada (TC), o que permite o planejamento com realização da osteotomia em modelo anatômico tridimensional, com o melhor entendimento da deformidade, aproximando-se da situação realística da cirurgia, o que torna o procedimento mais seguro, ágil e previsível. O objetivo do presente estudo é apresentar esta técnica e seu emprego em dois casos clínicos.

Distal radial fractures are very common. Vicious consolidation can occur in up to one third of these fractures, resulting in wrist pain, restricted movement, and, eventually, physical limitation or disability. The treatment of this condition consists in corrective osteotomy, which requires careful preoperative planning due to its three-dimensional complexity, especially in injuries with joint involvement. Recently, prototyping based on three-dimensional (3D) reconstruction of computed tomography (CT) scans has been used for osteotomy planning in a 3D anatomical model. It allows a better understanding of the deformity in a
Trabalho realizado pelo grupo de Cirurgia da Mão e Membro Superior, Departamento de Ortopedia e Traumatologia, Escola Paulista de Medicina, Universidade Federal de São Paulo, São Paulo, SP, Brasil. recebido

26 de Abril de 2020

aceito

06 de Julho de 2020

Publicado on-line

Fevereiro 10, 2021
DOI https://doi.org/ 10.1055/s-0040-1718510. ISSN 0102-3616.

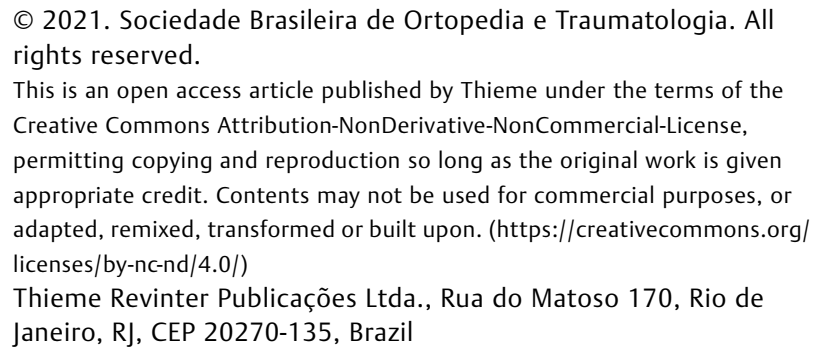

(c) 2021. Sociedade Brasileira de Ortopedia e Traumatologia. All rights reserved.

This is an open access article published by Thieme under the terms of the Creative Commons Attribution-NonDerivative-NonCommercial-License, permitting copying and reproduction so long as the original work is given appropriate credit. Contents may not be used for commercial purposes, or adapted, remixed, transformed or built upon. (https://creativecommons.org/ licenses/by-nc-nd/4.0/) Thieme Revinter Publicações Ltda., Rua do Matoso 170, Rio de Janeiro, RJ, CEP 20270-135, Brazil 
- three-dimensional printing realistic surgical approach, leading to safer, faster, and more predictable procedures. The aim of the present study is to present this technique and show its use in two clinical cases.

\section{Introdução}

Fraturas do terço distal do rádio estão entre as mais comuns do corpo, atingindo até $75 \%$ das fraturas de antebraço. ${ }^{1}$ Apresentam distribuição bimodal, acometendo jovens em sua maioria do sexo masculino decorrente de trauma de alta energia por um lado, e por outro, pacientes $>65$ anos, predominantemente do sexo feminino, com trauma de baixa energia, decorrente da fragilidade óssea-osteopenia. ${ }^{1,2}$ Um estudo epidemiológico realizado na Suécia, com 11,2 milhões de habitantes, entre os anos de 1999 a 2010, apontou incidência de 278 fraturas do terço distal do rádio a cada 100.000 indivíduos, com razão entre os sexos de 3 mulheres para cada homem. ${ }^{2}$ Em uma correlação com a cidade de São Paulo, com cerca de 12,2 milhões de habitantes, teríamos $>41$ mil fraturas no mesmo período; considerando-se que até $33 \%$ evoluam com consolidação viciosa, ${ }^{3,4}$ teríamos cerca de 13 mil pacientes, o que demonstra a importância de estudos para promover a prevenção e o tratamento desta condição clínica.

Ainda não há consenso em relação à melhor forma de tratamento das fraturas do rádio distal, ${ }^{3}$ e embora as técnicas cirúrgicas e implantes tenham uma evolução constante, as complicações com fraturas de rádio distal ainda persistem, sendo a artrose pós-traumática, a ruptura tendínea, a compressão do nervo mediano e a consolidação viciosa as mais freqüentes. ${ }^{3,4}$ A consolidação viciosa pode ocorrer em até $33 \%$ dos casos, ${ }^{4}$ sendo mais comum nos casos tratados de forma não cirúrgica.

As consolidações viciosas do rádio distal podem acarretar limitações funcionais e álgicas em diversos graus, segundo o tipo das deformidades. As deformidades angulares determinam um movimento compensatório anormal na articulação mediocárpica que evolui com instabilidade e consequente dor, limitação do movimento e artrose degenerativa do punho. As deformidades com encurtamento do rádio podem levar a impacto ulnocarpal e instabilidade da articulação radioulnar distal. Nas deformidades com degrau articular há grande probabilidade de evolução com alterações degenerativas do punho. Knirk et al. ${ }^{5}$ avaliaram o resultado funcional após tratamento de rádio distal em adultos jovens, e a congruência articular foi o fator crítico para o bom resultado funcional. Nos casos em que não se observou degrau articular, artrose pós-traumática ocorreu em $11 \%$ dos pacientes versus $91 \%$ em casos de não congruência articular. ${ }^{5}$ Estas alterações funcionais acarretam limitação funcional que podem significar a incapacidade laboral permanente do paciente.

As osteotomias corretivas para consolidação viciosa do rádio distal visam recuperar a anatomia óssea normal com o objetivo de melhora funcional e alívio da dor, além de prevenir a progressão de alterações degenerativas da articulação do punho. Muitas técnicas de osteotomia corretiva estão descritas devido à ampla variabilidade de apresentação das consolidações viciosas, porém o ponto comum para sua indicação é a presença de dor e de limitação funcional do paciente somado à ausência de artrose avançada na articulação radiocarpal, possibilitando um benefício clínico significativo com a correção cirúrgica. ${ }^{6}$

A análise clínica e radiológica são fundamentais para o bom desfecho da correção das deformidades, além das radiografias com incidências posteroanterior e lateral verdadeiro do punho acometido e contralateral, o emprego da tomografia computadorizada (TC) é essencial para avaliar o comprometimento articular e o planejamento cirúrgico, principalmente nas consolidações viciosas com envolvimento da articulação. ${ }^{7}$

Recentemente, a TC com reconstrução 3D para execução de prototipagem em modelo tridimensional utilizando o ácido polilático (PLA), permite o melhor entendimento da deformidade, além de ser de grande valia para o planejamento cirúrgico. $^{6,7} \mathrm{O}$ cuidadoso planejamento, que é realizado previamente ao procedimento cirúrgico no modelo 3D em dimensões reais às do paciente, permite ao cirurgião validar o tipo de implante a ser utilizado e prever as etapas e estratégias do procedimento cirúrgico. Essa abordagem proporciona tempo cirúrgico reduzido, melhor escolha e o posicionamento dos implantes, além de validar o local e a direção exatas da osteotomia necessária para a correção da deformidade. ${ }^{7,8}$ Há cerca de 2 anos, com apoio da Fundação de Amparo à Pesquisa do Estado de São Paulo (FAPESP), foi implantado o laboratório de prototipagem com impressão em 3D, o que permitiu que pudéssemos iniciar o desenvolvimento de estudos com utilização desta técnica que vamos descrever a seguir.

\section{Indicações e Contraindicações}

A impressão 3D em modelo prototipado (PLA) da consolidação viciosa de rádio distal durante o planejamento préoperatório é recomendada para todas as cirurgias corretivas de consolidação viciosa da porção distal do rádio devido à complexa distorção da anatomia que a condição clínica apresenta. Esta estratégia permite o melhor entendimento tridimensional e da real correção da deformidade em pacientes que apresentem deformidade anatômica e limitação funcional significativa.

As osteotomias corretivas para consolidação viciosa do rádio distal estão contraindicadas nos pacientes com baixa demanda de atividade diária, naqueles que tenham deformidade anatômica leve e que apresentem pouca restrição funcional; bem como nos pacientes com longa evolução da deformidade e que apresentem osteoartrite degenerativa radiocarpal. Assim, nas deformidades com incongruência articular ou extra-articular de longa evolução, recomendamos uma avaliação mais específica do grau de degeneração 
da cartilagem articular, utilizando-se da ressonância nuclear magnética (RNM) ou da artroscopia de punho; em vigência de degeneração articular grave, a correção com osteotomia está contraindicada.

\section{Técnica Pré-Operatória}

A TC bilateral dos punhos deve ser realizada com espaçamento entre os cortes de até $1 \mathrm{~mm}$ de espessura. $\mathrm{O}$ arquivo gerado no formato e padronização Digital Imaging and Communications in Medicine (DICOM, na sigla em inglês) é importado para um software de processamento e reconstrução 3D de imagens médicas (InVesalius versão 3.1.1, Centro de Tecnologia da Informação Renato Archer, Campinas, SP, Brasil). Este modelo tridimensional do rádio distal é exportado como arquivo Standart Tessellation Language / STereo-Lithography (STL, na sigla em inglês), e posteriormente o software Simplify3D (Simplify3D: Cincinnati, Ohio, USA) traduz as informações contidas no arquivo .stl em instruções para a impressora 3D. O material usado para impressão 3D é o ácido polilático (PLA).

Após a confecção dos protótipos do rádio distal com a deformidade e do lado contralateral normal, realizamos o planejamento da osteotomia corretiva de rádio distal. O posicionamento da placa, comprimento dos parafusos e local da osteotomia devem ser avaliados com o auxílio da fluoroscopia - arco em C, para a correta escolha de materiais para o procedimento cirúrgico.

A realização da programação cirúrgica com reconstrução 3D e modelo prototipado em PLA permite ao cirurgião o melhor entendimento da deformidade, planejar com perspectiva real o local exato da osteotomia e determinar o melhor tipo e especificações dos implantes a serem utilizados no procedimento cirúrgico. Deste modo, antecipa e otimiza as etapas da operação, o que torna o procedimento mais seguro, ágil e a correção da deformidade mais previsível. Este projeto de pesquisa foi analisado e aprovado pelo comitê de ética sob o número 9253251119.

\section{Casos Ilustrativos}

Caso 1-Paciente feminina, 46 anos de idade, sequela de fratura da porção distal do rádio esquerdo há 4 anos, tratada na ocasião com imobilização gessada, evolui com quadro de dor e limitação de flexão do punho esquerdo. Radiografia posteroanterior e perfil absoluto do punho esquerdo demonstrando consolidação viciosa (-Figura 1). Prototipagem a partir da tomografia computadorizada com reconstrução 3D do rádio distal bilateral permitiu o melhor entendimento da deformidade e planejamento cirúrgico (-Figura 2).

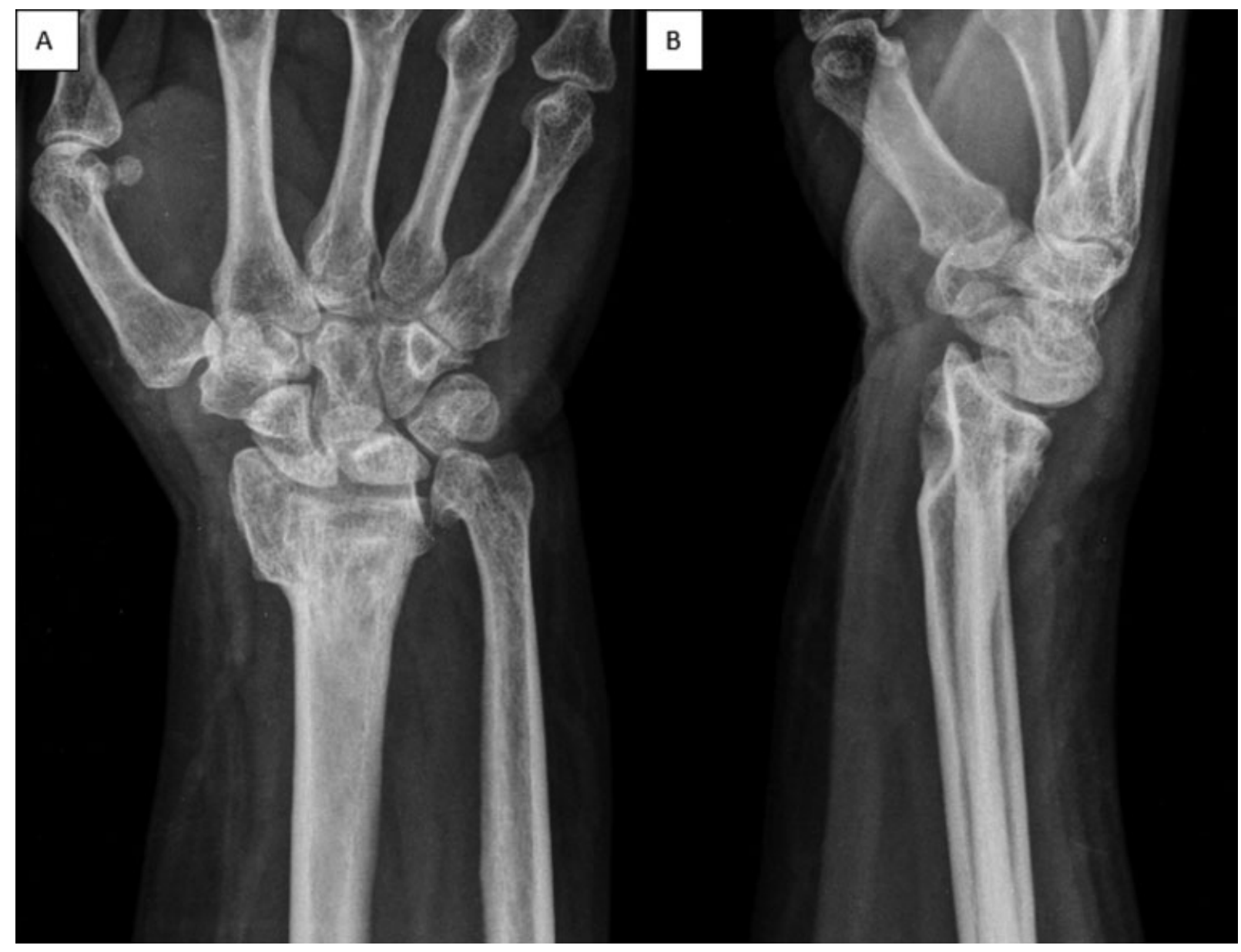

Fig. 1 Radiografia posteroanterior (A) e perfil absoluto (B) do punho esquerdo demonstrando consolidação viciosa com desvio dorsal, encurtamento do rádio e sequela com deformidade da cabeça da ulna, associado a instabilidade cárpica adaptativa. 


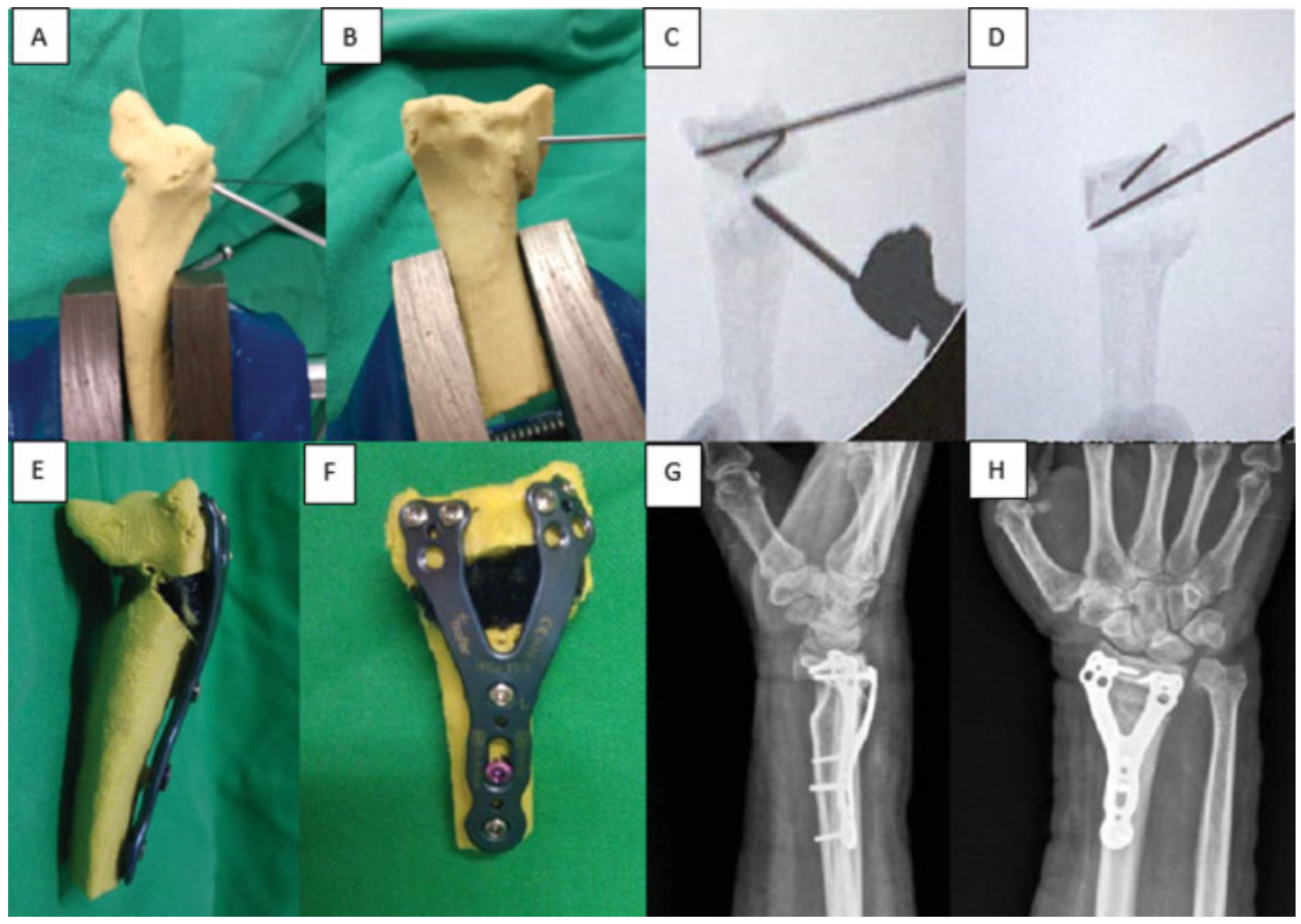

Fig. 2 Planejamento e cirurgia com prototipagem em modelo (PLA): colocação dos fios guia para osteotomia no modelo (A,B) e controle por escopia da osteotomia do modelo (C,D), prototipagem pós-osteotomia corretiva e fixação com placa dorsal (E,F). Imagens de controle radiográfico pós-operatório $(\mathrm{G}, \mathrm{H})$.

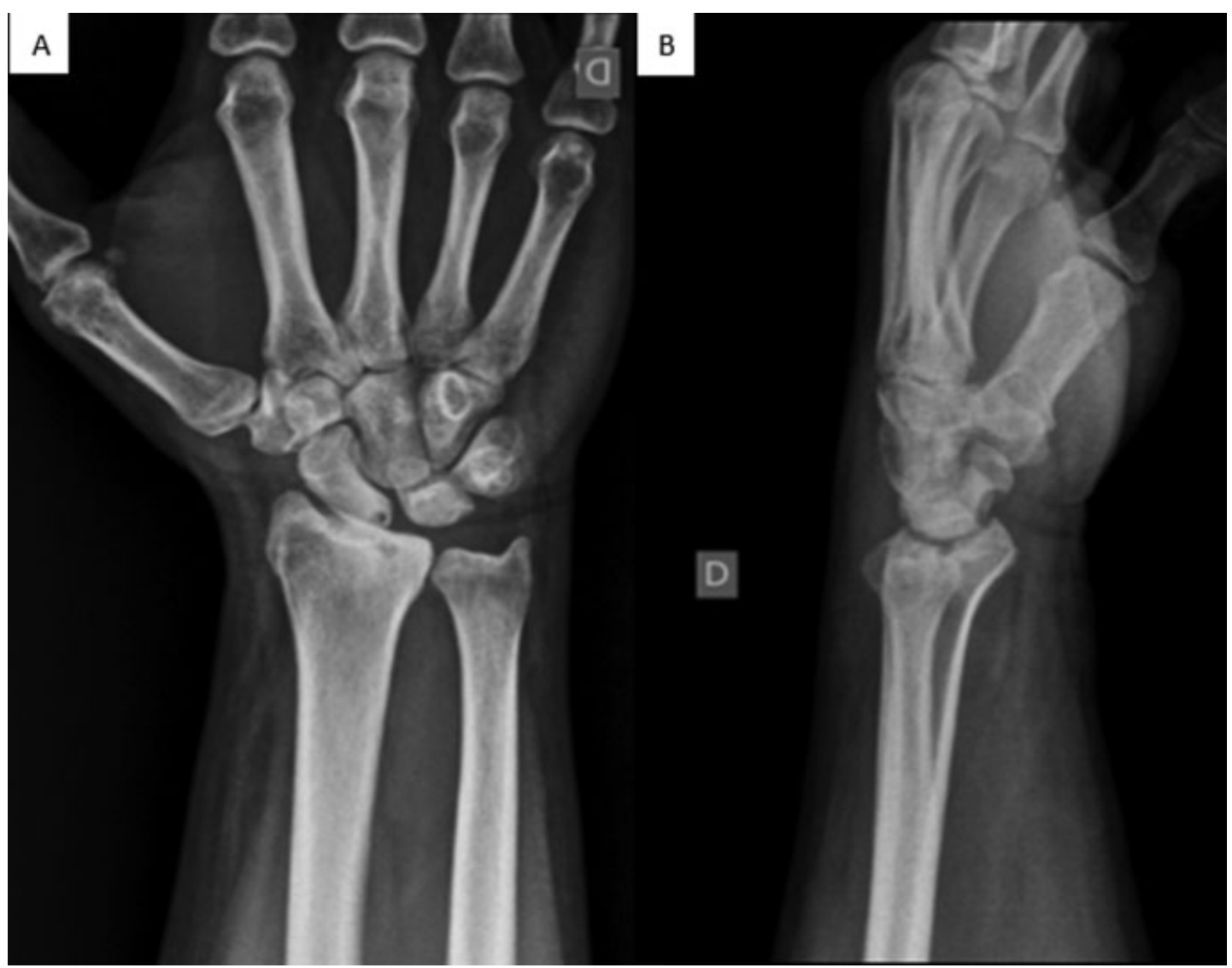

Fig. 3 Radiografias em posteroanterior (A) e perfil (B) da porção distal do rádio direito, consolidação viciosa com degrau articular radiocarpal e perda da inclinação volar normal do rádio. 


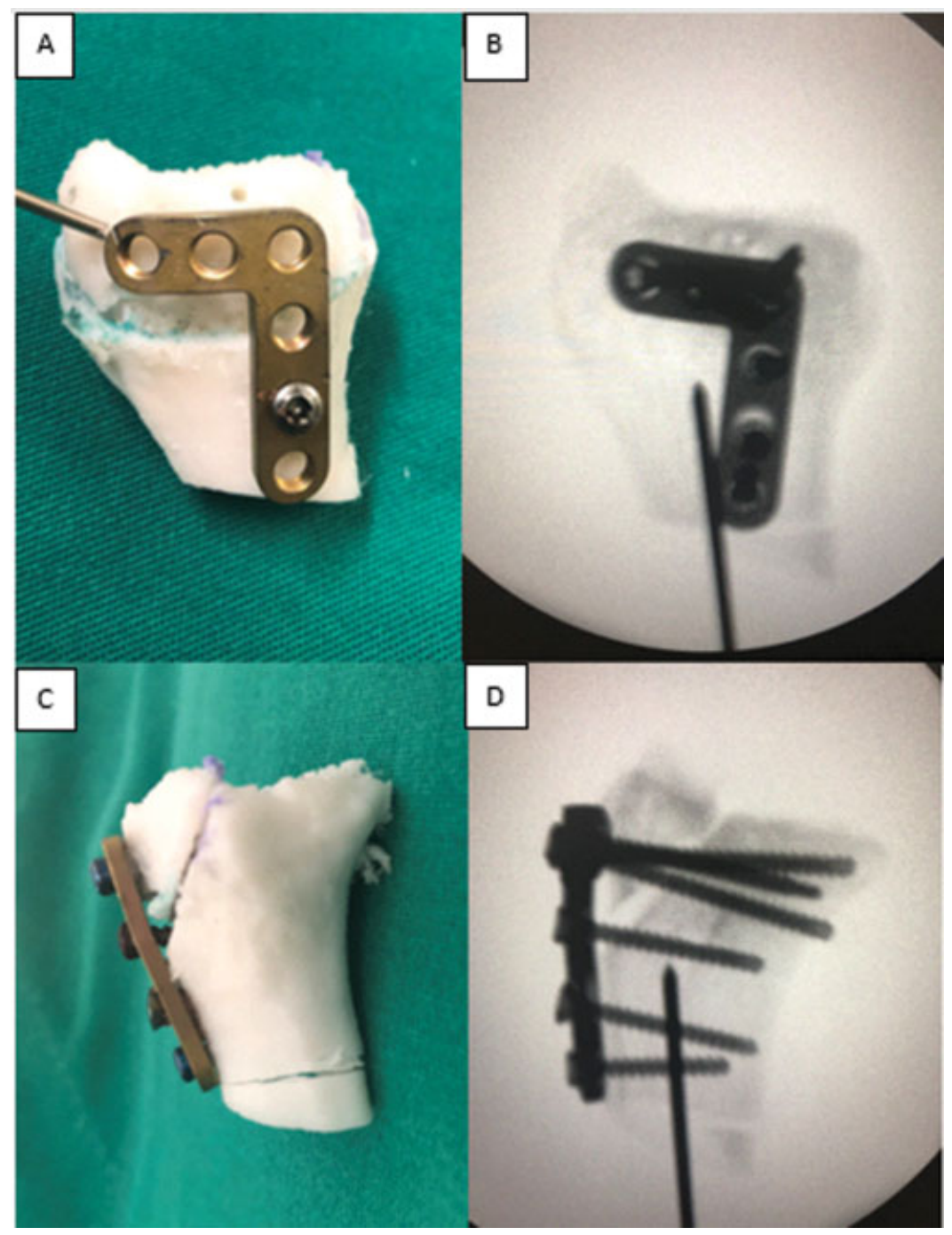

Fig. 4 Vista dorsal: planejamento da osteotomia corretiva articular da porção distal do rádio direito no modelo impresso (A), e imagem fluoroscópica pós-fixação da placa dorsal bloqueada de rádio distal (B). Vista lateral: osteotomia articular da porção distal do rádio e fixação com placa dorsal bloqueada no modelo impresso (C) e imagem fluoroscópica com correção dos parâmetros radiográficos (D).

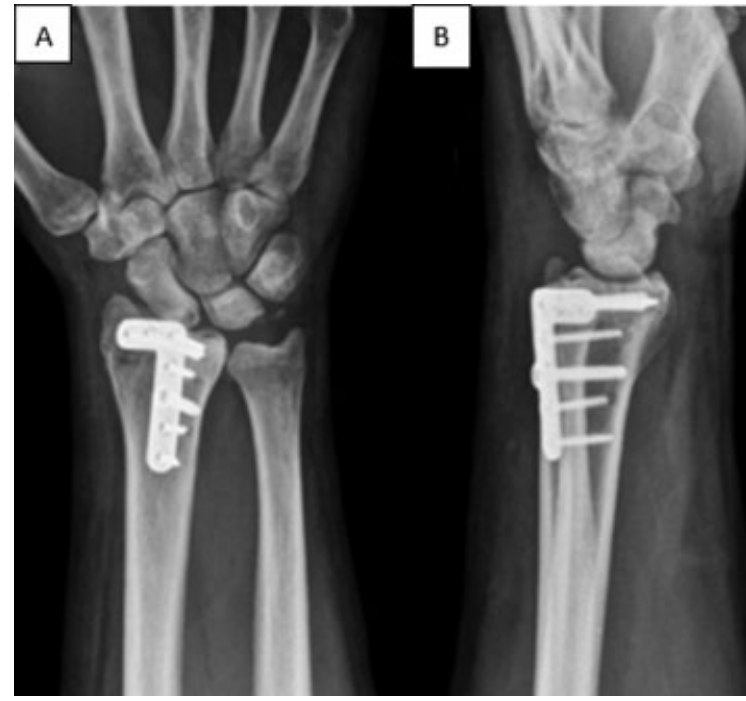

Fig. 5 Radiografia pós-operatória com 4 semanas de evolução, posteroanterior (A) e perfil (B). Redução do degrau articular e do alargamento da superfície articular do rádio, conforme realizado no planejamento pós-operatório em modelo PLA.
Caso 2-Paciente masculino, 47 anos de idade, pós-tratamento não cirúrgico de fratura da porção distal do rádio direito ocorrida há 4 meses. Nas radiografias do punho, evidencia-se consolidação viciosa com perda da inclinação volar e alargamento da superfície articular do rádio distal com degrau articular radiocarpal (-Figura 3). De forma semelhante ao caso anterior, imprimiu-se modelo em ácido polilático de ambos os rádios distais do paciente, onde foi esquematizado o ponto de osteotomia e correção da deformidade ( - Figuras 4 e 5 ).

\section{Discussão}

No começo da década de 80 , Charles Hull desenvolveu e conceitualizou a impressão em 3D, o que permitiu a criação de objetos a partir de deposição de materiais camada a camada. Desde então, avanços têm sido feitos na impressão em 3D, resultando em modelos com melhor resolução, produção mais rápida e a menor custo, além de ter permitido variedade maior de materiais para impressão. ${ }^{7}$

$\mathrm{O}$ uso da prototipagem em 3D na cirurgia ortopédica permite o melhor entendimento tridimensional da fratura 
ou consolidação viciosa. A possibilidade de visualizar e manipular os modelos, que são fidedignos à anatomia do paciente, ajuda na programação cirúrgica e na tomada de decisão intraoperatória.

\section{Considerações Finais}

Nos casos de consolidação viciosa da porção distal do rádio, a impressão do modelo a partir da reconstrução em 3D da tomografia computadorizada auxilia o cirurgião na correta escolha de implante, direção e local da osteotomia corretiva. Esse planejamento pré-operatório poupa tempo cirúrgico, o que está associado a um menor índice de complicações e resultado funcional mais favorável.

Financiamento

O presente estudo foi desenvolvido no laboratório de Prototipagem com Impressão em 3D com recursos provenientes do projeto regular - Fundação de Amparo à Pesquisa do Estado de São Paulo - FAPESP número 17/26283-0.

Conflito de Interesses

Os autores não têm nenhum conflito de interesse a declarar.

\section{Referências}

1 Chung KC, Spilson SV. The frequency and epidemiology of hand and forearm fractures in the United States. J Hand Surg Am 2001; 26(05):908-915

2 Jerrhag D, Englund M, Karlsson MK, Rosengren BE. Epidemiology and time trends of distal forearm fractures in adults - a study of 11.2 million person-years in Sweden. BMC Musculoskelet Disord 2017;18(01):240

3 Belloti JC, Santos JBG, Atallah AN, Albertoni WM, Faloppa F. Fractures of the distal radius (Colles' fracture). Sao Paulo Med J 2007;125(03):132-138

4 Cole JM, Obletz BE. Comminuted fractures of the distal end of the radius treated by skeletal transfixation in plaster cast: an endresult study of thirty-three cases. J Bone Joint Surg Am 1966;48 (05):931-945

5 Knirk JL, Jupiter JB. Intra-articular fractures of the distal end of the radius in young adults. J Bone Joint Surg Am 1986;68(05):647-659

6 Van Cauwelaert de Wyels J, De Smet L. Corrective osteotomy for malunion of the distal radius in young and middle-aged patients: an outcome study. Chir Main 2003;22(02):84-89

7 Stockmans F, Dezillie M, Vanhaecke J. Accuracy of 3D virtual planning of corretive osteotomies of the distal radius. J Wrist Surg 2013;2(04):306-314

8 Walenkamp MM, de Muinck Keizer RJ, Dobbe JG, et al. Computerassisted 3D planned corrective osteotomies in eight malunited radius fractures. Strateg Trauma Limb Reconstr 2015;10(02): 109-116 